\title{
Numerical Analysis of Inhomogeneous Dielectric Waveguide Using Periodic Fourier Transform
}

\author{
M. Moradian and M. Khalaj-Amirhosseini \\ Received 22 September 2007; Accepted 24 December 2007 \\ Recommended by Yue Ping Zhang \\ A general method is introduced to obtain the propagation constants of the inhomogeneous dielectric waveguide. The periodic \\ Fourier transform is applied to the normalized Maxwell's equations and makes the field components periodic. Then they are ex- \\ panded in Fourier series. Finally, the trapezoidal rule is applied to approximate the convolution integral which leads to a set of \\ coupled second-order differential equations that can be solved as an eigenvalue-eigenvector problem. The normalized propagation \\ constant can be obtained as the square roots of the eigenvalues of the coefficient matrices. The proposed method is applied to \\ the dielectric waveguide with a two-layered dielectric profile in the transverse direction, and the first four-confined TE modes are \\ obtained. The propagation constants for the mentioned dielectric waveguide are also derived analytically and are then compared \\ with those derived by the proposed method. Comparison of results shows the efficacy of the proposed method.
}

Copyright (c) 2007 M. Moradian and M. Khalaj-Amirhosseini. This is an open access article distributed under the Creative Commons Attribution License, which permits unrestricted use, distribution, and reproduction in any medium, provided the original work is properly cited.

\section{INTRODUCTION}

Dielectric waveguide with various or inhomogeneous dielectric layers profile has been addressed in many research literatures. The accurate determination of propagation characteristic for such a transmission media is essential in design and optimization of various types of optical and millimeter wave devices. Several computation methods have been proposed to obtain the propagation characteristic of the planar graded-index dielectric waveguide. Some approximate analytical methods such as WKB [1], perturbation method [2], IBMOM [3], and GIBMOM [4] are effective for computing the propagation characteristic of these types of waveguides. Other methods that use computationally intensive methods such as sine/cosine expansion methods $[5,6]$, finite difference methods [7], and finite element methods [8] are also effective for this purpose. A proper method for computing the propagation characteristic of inhomogeneous dielectric waveguide is presented, which is based on the periodic Fourier transform. The periodic Fourier transform has been established in several literatures. In [9] the periodic Fourier transform was proposed to investigate the scattering problems in a periodic structure with finite extent, while in [10] it was used to analyze a series of transition in dielectric waveguide. In this paper, a method in [10] will be developed to determine the propagation constants of inhomogeneous dielectric waveguides. To show the validity of the proposed method, the method is applied to a two-layered dielectric waveguide. But, the application of the proposed method is not essentially restricted to the multilayer dielectric waveguide profile, and can be easily applied to any inhomogeneous dielectric waveguide profiles.

\section{WAVE PROPAGATION IN GRADE-INDEX WAVEGUIDE}

In this section, the propagation constant for the TE waves in inhomogeneous slab dielectric waveguide is derived. Cartesian coordinates $O x y z$ is introduced and oriented such that $\mathrm{Oz}$ is parallel to the direction of propagation and $O x$ is in the direction of the dielectric profile variation as shown in Figure 1. The geometry and the fields are also uniform and have no variation in the $y$-direction. The value of dielectric constant changes in the transverse direction as a function of $x$. The dielectric is assumed to be linear, isotropic and its permeability set is equal to the free space permeability. The fields are time harmonic and complex variables. They have only $x$ and $z$ dependency. The Electric field intensity $\mathbf{E}$, Magnetic field intensity $\mathbf{H}$, and Electric flux density $\mathbf{D}$ are normalized by $\sqrt[4]{\mu_{0} / \varepsilon_{0}}, \sqrt[4]{\varepsilon_{0} / \mu_{0}}$, and $\varepsilon_{0} \sqrt[4]{\mu_{0} / \varepsilon_{0}}$, respectively.In these relations, $\varepsilon_{0}$ and $\mu_{0}$ are the permittivity and permeability of free space, respectively. 


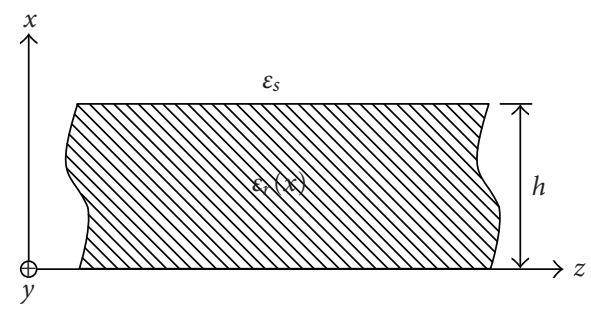

FIGURE 1: Cross section of the investigated inhomogeneous dielectric profile.

The dielectric constant can be decomposed into two parts, namely, constant and nonconstant parts and expressed as

$$
\varepsilon(x)=\varepsilon_{s}+\varepsilon_{\mathrm{nc}}(x) .
$$

The reciprocal of relative permittivity distribution is denoted by $\eta(x)=1 / \varepsilon(x)$ and decomposes as

$$
\eta(x)=\eta_{s}+\eta_{\mathrm{nc}}(x) .
$$

The first part is equal to $1 / \varepsilon_{s}$, while the last part is not equal to $1 / \varepsilon_{\mathrm{nc}}(x)$ since $\varepsilon_{\mathrm{nc}}(x)$ is zero in the region which is filled with dielectric waveguide slab.

Although many relations which are mentioned here are the same as those in [10], they will be mentioned here for simplicity.

From normalized Maxwell's equations, one has the following relations:

$$
\begin{gathered}
\frac{\partial}{\partial z} H_{x}(x, z)-\frac{\partial}{\partial x} H_{x}(x, z)=-i k_{0} D_{y}(x, z), \\
\frac{\partial}{\partial z} E_{y}(x, z)=-i k_{0} H_{x}(x, z), \\
\frac{\partial}{\partial x} E_{y}(x, z)=i k_{0} H_{z}(x, z) .
\end{gathered}
$$

In these equations, $k_{0}$ is the wave number in free space. The dielectric constant has inhomogeneous profile in the transverse $x$ direction and the required constitutive relation is in the form

$$
D_{y}(x, z)=\varepsilon(x) E_{y}(x, z) .
$$

Applying the periodic Fourier transform to (3), the following equations will be achieved:

$$
\begin{aligned}
& \frac{\partial}{\partial z} \tilde{H}_{x}(x, \alpha, z)-\left(\frac{\partial}{\partial x}+i \alpha\right) \tilde{H}_{x}(x, \alpha, z)=-i k_{0} \widetilde{D}_{y}(x, \alpha, z), \\
& \frac{\partial}{\partial z} \widetilde{E}_{y}(x, \alpha, z)=-i k_{0} \tilde{H}_{x}(x, \alpha, z), \\
& \left(\frac{\partial}{\partial x}+i \alpha\right) \widetilde{E}_{y}(x, \alpha, z)=i k_{0} \tilde{H}_{z}(x, \alpha, z), \\
& \tilde{D}_{y}(x, \alpha, z) \\
& \quad=\varepsilon_{s} \widetilde{E}_{y}(x, \alpha, z)+\frac{d}{2 \pi} \int_{0}^{2 \pi / d} \widetilde{\varepsilon}_{\mathrm{nc}}(x, \alpha-\xi) \widetilde{E}_{y}(x, \xi, z) d \xi .
\end{aligned}
$$

All of the transformed relations are periodic in terms of $x$, because of the periodic nature of periodic Fourier transform. So they can be approximately expanded in the truncated Fourier series. By introducing the $(2 N+1) \times 1$ column matrices which have the Fourier series coefficients of all the electromagnetic fields components, the equations yield the following relations:

$$
\begin{gathered}
\frac{\partial}{\partial z} \tilde{h}_{x}(\alpha, z)-i k_{0} X(\alpha) \tilde{h}_{x}(\alpha, z)=-i k_{0} \tilde{d}_{y}(\alpha, z) \\
\frac{\partial}{\partial z} \tilde{e}_{y}(\alpha, z)=-i k_{0} \tilde{h}_{x}(\alpha, z) \\
X(\alpha) \tilde{e}_{y}(\alpha, z)=i k_{0} \tilde{h}_{z}(\alpha, z) \\
\tilde{d}_{y}(\alpha, z)=\varepsilon_{s} \tilde{e}_{y}(\alpha, z)+\frac{d}{2 \pi} \int_{0}^{2 \pi / d}\left[\left[\tilde{\varepsilon}_{\mathrm{nc}}\right]\right](\alpha-\xi) \tilde{e}_{y}(\xi, z) d \xi
\end{gathered}
$$

In the above equations, the matrices $X(\alpha)$ and $\left[\left[\tilde{\mathcal{E}}_{\mathrm{nc}}\right]\right](\alpha)$ are derived by

$$
\begin{gathered}
(X(\alpha))_{n, m=} \delta_{n, m}\left(\frac{\alpha}{k_{0}}+n \frac{\lambda_{0}}{d}\right), \\
\left(\left[\left[\widetilde{\varepsilon}_{\mathrm{nc}}\right]\right](\alpha)\right)_{n, m=} \widetilde{\varepsilon}_{\mathrm{nc}, n-m}(\alpha) .
\end{gathered}
$$

Here, $\delta_{n, m}$ is the Kronecker delta and $\widetilde{\varepsilon}_{c, n}(\alpha)$ is the $n$ th-order Fourier coefficients of $\widetilde{\varepsilon}_{c}(x, \alpha)$ and it is given by

$$
\begin{aligned}
\widetilde{\mathcal{E}}_{\mathrm{nc}, n}(\alpha) & =\frac{1}{d} \int_{0}^{d} \widetilde{\mathcal{E}}_{\mathrm{nc}}(x, \alpha) e^{-\mathrm{in}(2 \pi / d) x} d x \\
& =\frac{1}{d} \int_{-\infty}^{\infty} \widetilde{\varepsilon}_{\mathrm{nc}}(x) e^{-i(\alpha+n(2 \pi / d)) x} d x .
\end{aligned}
$$

Taking $L+1$ equispace sample points between $[0,2 \pi / d]$ and approximate the convolution integral by applying the trapezoidal rule to it, the following will eventually arrive:

$$
\begin{aligned}
& \tilde{d}_{y}\left(\alpha_{l}, z\right) \\
& =\varepsilon_{s} \tilde{e}_{y}\left(\alpha_{l}, z\right)+\frac{1}{2 L}\left(\left[\left[\widetilde{\varepsilon}_{\mathrm{nc}}\right]\right]\left(\alpha_{l}\right) \tilde{e}_{y}\left(\alpha_{0}, z\right)\right. \\
& \left.\quad+2 \sum_{m=1}^{L-1}\left[\left[\tilde{\varepsilon}_{\mathrm{nc}}\right]\right]\left(\alpha_{l-m}\right) \tilde{e}_{y}\left(\alpha_{m}, z\right)+\left[\left[\tilde{\varepsilon}_{\mathrm{nc}}\right]\right]\left(\alpha_{l-L}\right) \tilde{e}_{y}\left(\alpha_{L}, z\right)\right) .
\end{aligned}
$$

Introduce $(L+1) \times(2 N+1) \times 1$ column matrices to express the discretized Fourier coefficients at $(L+1)$ sample points by

$$
\tilde{e}_{y}(\mathrm{z})=\left(\begin{array}{c}
\tilde{e}_{y}\left(\alpha_{0}, z\right) \\
\vdots \\
\tilde{e}_{y}\left(\alpha_{L}, z\right)
\end{array}\right) .
$$


So (6)-(8) can be rewritten in the form

$$
\begin{gathered}
\frac{d}{d z} \bar{h}_{x}(z)-i k_{0} \bar{X} \cdot \bar{h}_{z}(z)=-i k_{0} \bar{d}_{y}(z), \\
\frac{d}{d z} \bar{e}_{y}(z)=-i k_{0} \bar{h}_{x}(z), \\
\bar{X} \cdot \bar{e}_{y}(y)=\bar{h}_{z}(z), \\
\bar{d}_{y}(z)=[[[\varepsilon]]] \bar{e}_{y}(z),
\end{gathered}
$$

with

$$
\begin{aligned}
& \bar{X}(\alpha)=\left[\begin{array}{cccc}
X\left(\alpha_{0}\right) & 0 & \cdots & 0 \\
0 & \ddots & \ddots & \vdots \\
\vdots & \ddots & \ddots & 0 \\
0 & \cdots & 0 & X\left(\alpha_{L}\right)
\end{array}\right] \\
& {[[[\varepsilon]]]=\varepsilon_{s} I+\left[\left[\left[\varepsilon_{\mathrm{nc}}\right]\right]\right],} \\
& {\left[\left[\left[\varepsilon_{\mathrm{nc}}\right]\right]\right]=\frac{1}{2 L}\left(\begin{array}{ccccc}
a^{\prime} & a^{\prime \prime} & \cdots & a^{\prime \prime \prime} & a^{\prime \prime \prime \prime} \\
\vdots & \vdots & \ddots & \vdots & \vdots \\
q^{\prime} & q^{\prime \prime} & \cdots & q^{\prime \prime \prime} & q^{\prime \prime \prime \prime}
\end{array}\right)}
\end{aligned}
$$

where $a^{\prime}$ denotes $\left[\left[\widetilde{\varepsilon}_{\mathrm{nc}}\right]\right]\left(\alpha_{0}\right), a^{\prime \prime}$ denotes $2\left[\left[\widetilde{\varepsilon}_{\mathrm{nc}}\right]\right]\left(\alpha_{-1}\right), a^{\prime \prime \prime}$ denotes $2\left[\left[\widetilde{\varepsilon}_{\mathrm{nc}}\right]\right]\left(\alpha_{-L+1}\right), a^{\prime \prime \prime \prime}$ denotes $\left[\left[\widetilde{\varepsilon}_{\mathrm{nc}}\right]\right]\left(\alpha_{-L}\right), q^{\prime}$ denotes $\left[\left[\tilde{\varepsilon}_{\mathrm{nc}}\right]\right]\left(\alpha_{L}\right), q^{\prime \prime}$ denotes $2\left[\left[\tilde{\varepsilon}_{\mathrm{nc}}\right]\right]\left(\alpha_{L-1}\right), q^{\prime \prime \prime}$ denotes $2\left[\left[\widetilde{\varepsilon}_{\mathrm{nc}}\right]\right]\left(\alpha_{1}\right)$, and $q^{\prime \prime \prime \prime}$ denotes $\left[\left[\widetilde{\varepsilon}_{\mathrm{nc}}\right]\right]\left(\alpha_{0}\right)$.

Finally, from (15), one can obtain the following secondorder differential equation as

$$
\frac{d^{2}}{d z^{2}} \tilde{e}_{y}(z)=-k_{0}^{2} C_{e} \tilde{e}_{y}(z)
$$

with

$$
C_{e}=[[[\varepsilon]]]-\bar{X}^{2}
$$

Similar to the TM modes, the normalized Maxwell's equations and the constitutive relations are as follows:

$$
\begin{aligned}
& \frac{\partial}{\partial z} E_{x}(x, z)-\frac{\partial}{\partial x} E_{z}(x, z)=i k_{0} H_{y}(x, z), \\
& \frac{\partial}{\partial x} H_{y}(x, z)=i k_{0} D_{x}(x, z), \\
& \frac{\partial}{\partial x} H_{y}(x, z)=-i k_{0} D_{y}(x, z), \\
& D_{x}(x, z)=\varepsilon(x) E_{x}(x, z), \\
& D_{z}(x, z)=\varepsilon(x) E_{z}(x, z) .
\end{aligned}
$$

Following the same procedure with the TE case, we obtain the following relations

$$
\begin{gathered}
\frac{d}{d z} \bar{e}_{x}(z)-i k_{0} \bar{X} \bar{e}_{z}(z)=i k_{0} \bar{h}_{y}(z), \\
\frac{d}{d z} \bar{h}_{y}(z)=i k_{0} \bar{d}_{x}(z), \\
\bar{X} \bar{h}_{y}(z)=-\bar{d}_{z}(z), \\
\bar{d}_{x}(z)=[[[\eta]]]^{-1} \bar{e}_{x}(z), \\
\bar{d}_{z}(z)=[[[\varepsilon]]] \bar{e}_{z}(z) .
\end{gathered}
$$

The expression of $[[[\eta]]]$ is driven just by replacing the notation $\varepsilon$ by $\eta$ in (11), (12), (17), and (18).

From (26), one can obtain another coupled differential equation as

$$
\frac{d^{2}}{d z^{2}} \bar{h}_{y}(z)=-k_{0}^{2} C_{h} \bar{h}_{y}(z)
$$

with

$$
C_{h}=[[[\eta]]]^{-1}\left(I-\bar{X}[[[\varepsilon]]]^{-1} \bar{X}\right) .
$$

The propagation constant for TE and TM modes can be obtained by solving coupled second-order differential equations (19) and (27) as an eigenvalue-eigenvector problem. The following example demonstrates how to use this method to find the propagation constant for a typical dielectric waveguide with two-layered dielectric profile.

\section{DESIGN EXAMPLE}

In this section, the proposed method is applied to a dielectric waveguide with a two-layered profile [11]. The profile of dielectric waveguide has the following form:

$$
\varepsilon_{r}= \begin{cases}1 & x<0 \\ 6.25 & 0 \leq x \leq \lambda_{0} \\ 2.25 & 0<x \leq 2 \lambda_{0} \\ 1 & x>0\end{cases}
$$

The height of the mentioned dielectric waveguide is equal to $2 \lambda_{0}, \lambda_{0}$ is the wavelength in free space. The first layer has the dielectric constant equal to 6.25 and the second one has the dielectric constant equal to 2.25.

Table 1 shows the exact normalized propagation constants for four-confined TE mode in this waveguide. These normalized propagation constants are derived analytically [11]. Normalized propagation constants for the mentioned waveguide are also derived by the proposed method and by selecting the number of integration segments and truncation order equal to 30 and 20, respectively. Figure 2 shows the relative error rate of the obtained propagation constants versus the truncated order $N$ and with the fictitious periods $d=3 \lambda_{0}$. Comparison of the numerical results shows the convergence of the propagation constants versus the truncation 
TABLE 1: The exact normalized propagation constant for four-confined mode in two-layered dielectric waveguide.

\begin{tabular}{cccc}
\hline$\beta_{0}$ & $\beta_{1}$ & $\beta_{2}$ & $\beta_{3}$ \\
\hline 2.46190937255032 & 2.34488648040691 & 2.13982061988205 & 1.82913990191955 \\
\hline
\end{tabular}

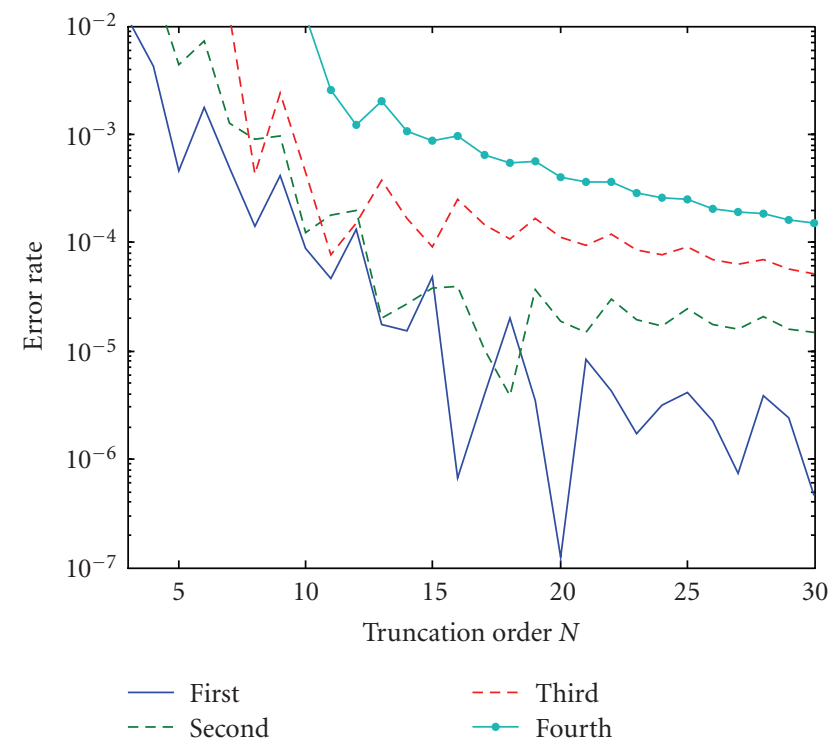

FIGURE 2: Error rate of the four-confined TE mode versus truncation order $N$.

order $N$. The values of error rates are also increased as the order of TE modes is increased. Figure 3 shows the error rate for first TE mode versus truncation order $N$ and the fictitious periods $d=0.2 \lambda_{0}, 0.5 \lambda_{0}, \lambda_{0}, 2 \lambda_{0}$, and $5 \lambda_{0}$. The number of integration segments is also equal to 30 in Figure 3 . The convergence of error rate for the fictitious periods $d=0.2 \lambda_{0}$, $0.5 \lambda_{0}$, and $\lambda_{0}$ is fast, while for other fictitious periods, there is a slow convergence and they have worse accuracy limit. Figure 4 shows the error rate for the first TE mode versus number of integration segments and with the fictitious periods $d=0.2 \lambda_{0}, 0.5 \lambda_{0}, \lambda_{0}, 2 \lambda_{0}$, and $5 \lambda_{0}$. The number of truncation order is also selected equal to 15 during the numerical procedure. As Figure 4 shows, the convergence of error rate for the fictitious periods $d=\lambda_{0}, 2 \lambda_{0}$ and $5 \lambda_{0}$ is much faster than the fictitious periods $d=0.2 \lambda_{0}$ and $0.5 \lambda_{0}$. It shows also that increasing the number of integration segments after a certain number will no longer alter the error rate. While for small value of this number and for the fictitious periods $d=0.2 \lambda_{0}$ and $0.5 \lambda_{0}$, there is a really worse accuracy limit. So by selecting a large fictitious period, the convergence of error rate with respect to $L$ becomes better, while the convergence of the relative error rate with respect to $N$ becomes worse.

\section{CONCLUSION}

In this paper, the periodic Fourier transform has been applied for finding the propagation constant of inhomogeneous dielectric waveguides. The proposed method is straightforward and does not need any boundary condition. The normalized field quantities became periodic by apply-

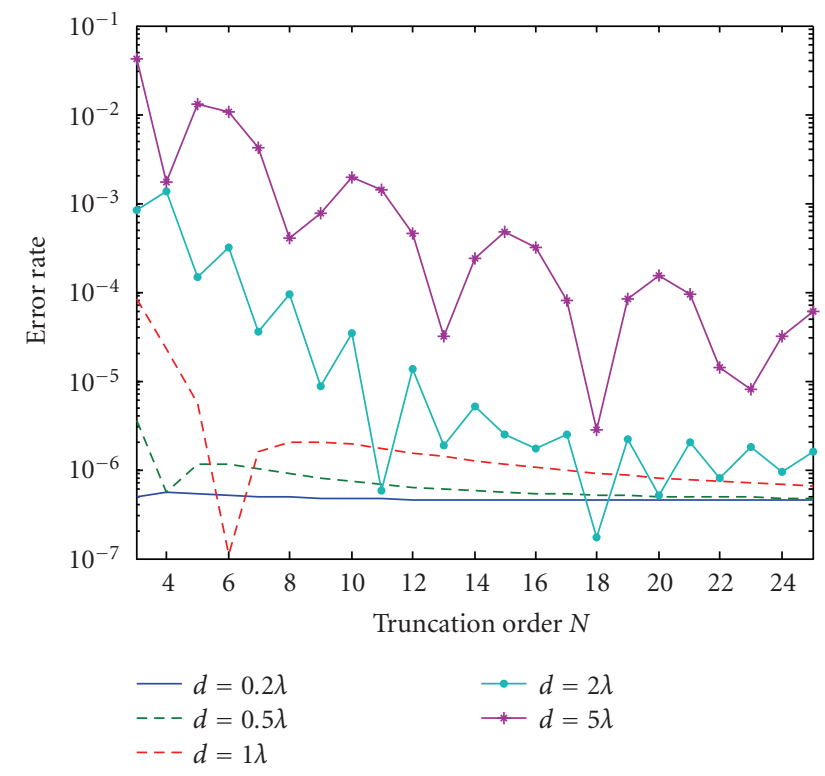

FIGURE 3: Error rate of the fundamental TE mode versus truncation order $N$.

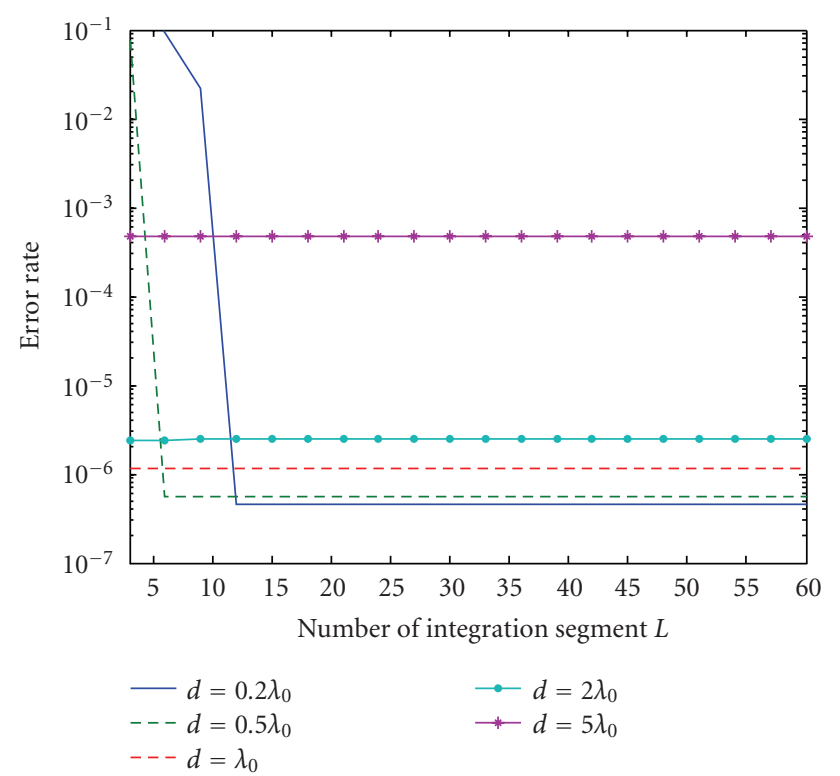

FIGURE 4: Error rate of the fundamental TE mode versus number of integration segment $L$.

ing the periodic Fourier transform to them. First, the truncation order Fourier transform of field quantity was derived. Next, by applying the trapezoidal rule to approximate the convolution integral, a set of coupled second-order differential equations was found. Then they were solved as an 
eigenvalue-eigenvector problem for finding the propagation constant of an inhomogeneous dielectric waveguide. To validate, a two-layered dielectric waveguide was selected as an objective and propagation constant for four-confined TE was computed by the proposed method. There is a good agreement between the results of the proposed method and those from the literature.

\section{REFERENCES}

[1] R. Srivastava, C. Kao, and R. Ramaswamy, "WKB analysis of planar surface waveguides with truncated index profiles," Journal of Lightwave Technology, vol. 5, no. 11, pp. 1605-1609, 1987.

[2] A. Kumar, K. Thyagarajan, and A. Ghatak, "Modes in inhomogeneous slab waveguides," IEEE Journal of Quantum Electronics, vol. 10, no. 12, pp. 902-904, 1974.

[3] A. Weisshaar, "Impedance boundary method of moments for accurate and efficient analysis of planar graded-index optical waveguides," Journal of Lightwave Technology, vol. 12, no. 11, pp. 1943-1951, 1994.

[4] J. Li and A. Weisshaar, "Generalised impedance boundary method of moments for multilayer graded-index dielectric waveguide structures," IEE Proceedings: Optoelectronics, vol. 143, no. 3, pp. 167-172, 1996.

[5] Y. Tu, I. C. Goyal, and R. L. Gallawa, "Analyzing integrated optical waveguides: a comparison of two new methods," Applied Optics, vol. 29, no. 36, pp. 5313-5315, 1990.

[6] A. Weisshaar and V. K. Tripathi, "Modal analysis of step discontinuities in graded-index dielectric slab waveguides," Journal of Lightwave Technology, vol. 10, no. 5, pp. 593-602, 1992.

[7] K. Bierwirth, N. Schulz, and F. Arndt, "Finite difference analysis of rectangular dielectric waveguide structures," IEEE Transactions on Microwave Theory and Techniques, vol. 34, no. 11, pp. 1104-1114, 1986.

[8] B. Rahman and J. B. Davies, "Finite-element solution of integrated optical waveguides," Journal of Lightwave Technology, vol. 2, no. 5, pp. 682-688, 1984.

[9] J. Nakayama, "Periodic Fourier transform and its application to wave scattering from a finite periodic surface," IEICE Transactions on Electronics, vol. E83-C, no. 3, pp. 481-487, 2000.

[10] K. Watanabe and K. Kuto, "Numerical analysis of optical waveguides based on Periodic Fourier transform," Progress in Electromagnetics Research, vol. 64, pp. 1-21, 2006.

[11] P. Yeh, Optical Waves in Layered Media, John Wiley \& Sons, New York, NY, USA, 2005.

\section{AUTHOR CONTACT INFORMATION}

M. Moradian: Department of Electrical Engineering, Iran

University of Science and Technology Narmak, Tehran 16844, Iran; m_moradian@ee.iust.ac.ir

M. Khalaj-Amirhosseini: Department of Electrical Engineering, Iran University of Science and Technology Narmak, Tehran 16844, Iran; khalaja@iust.ac.ir 

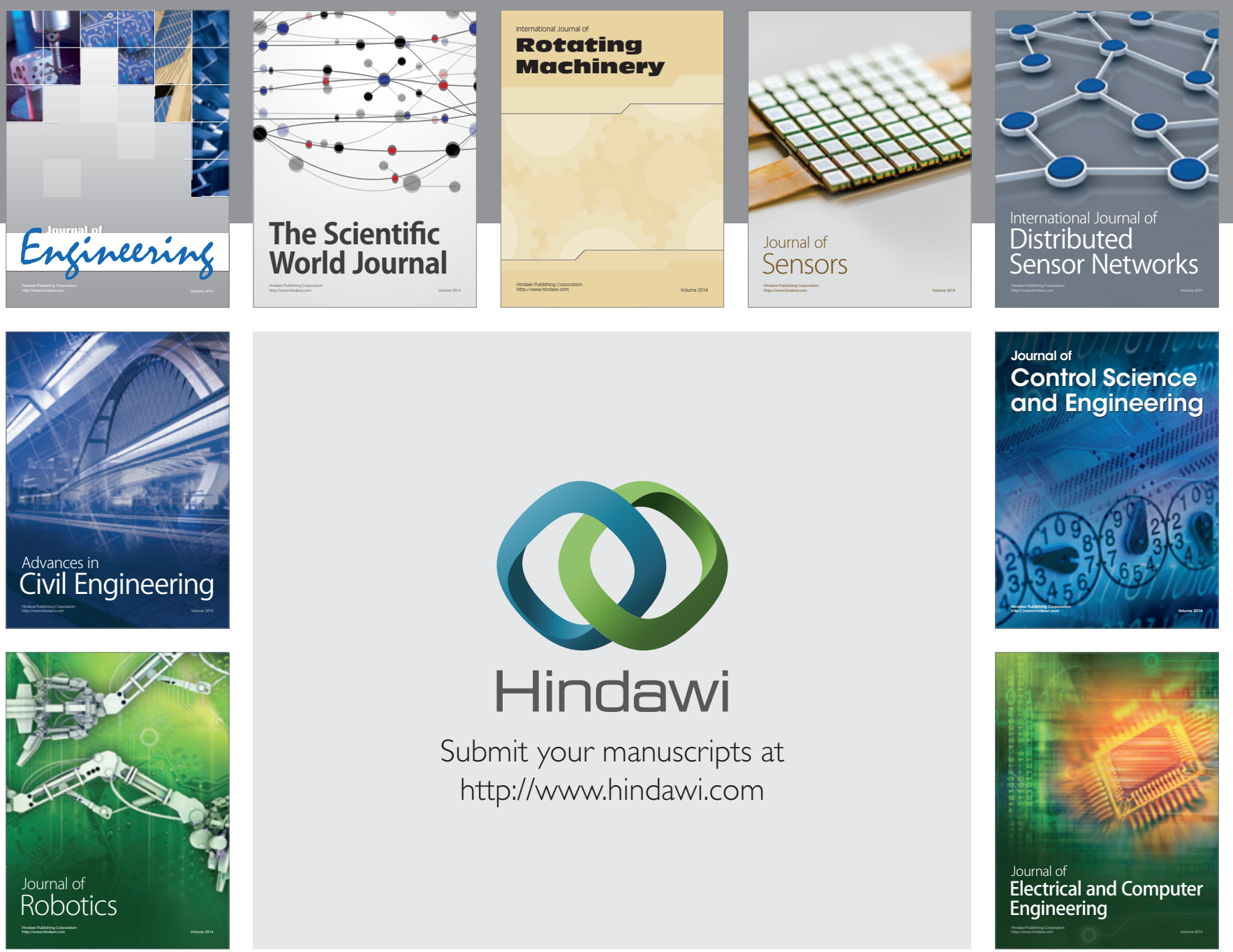

Submit your manuscripts at

http://www.hindawi.com
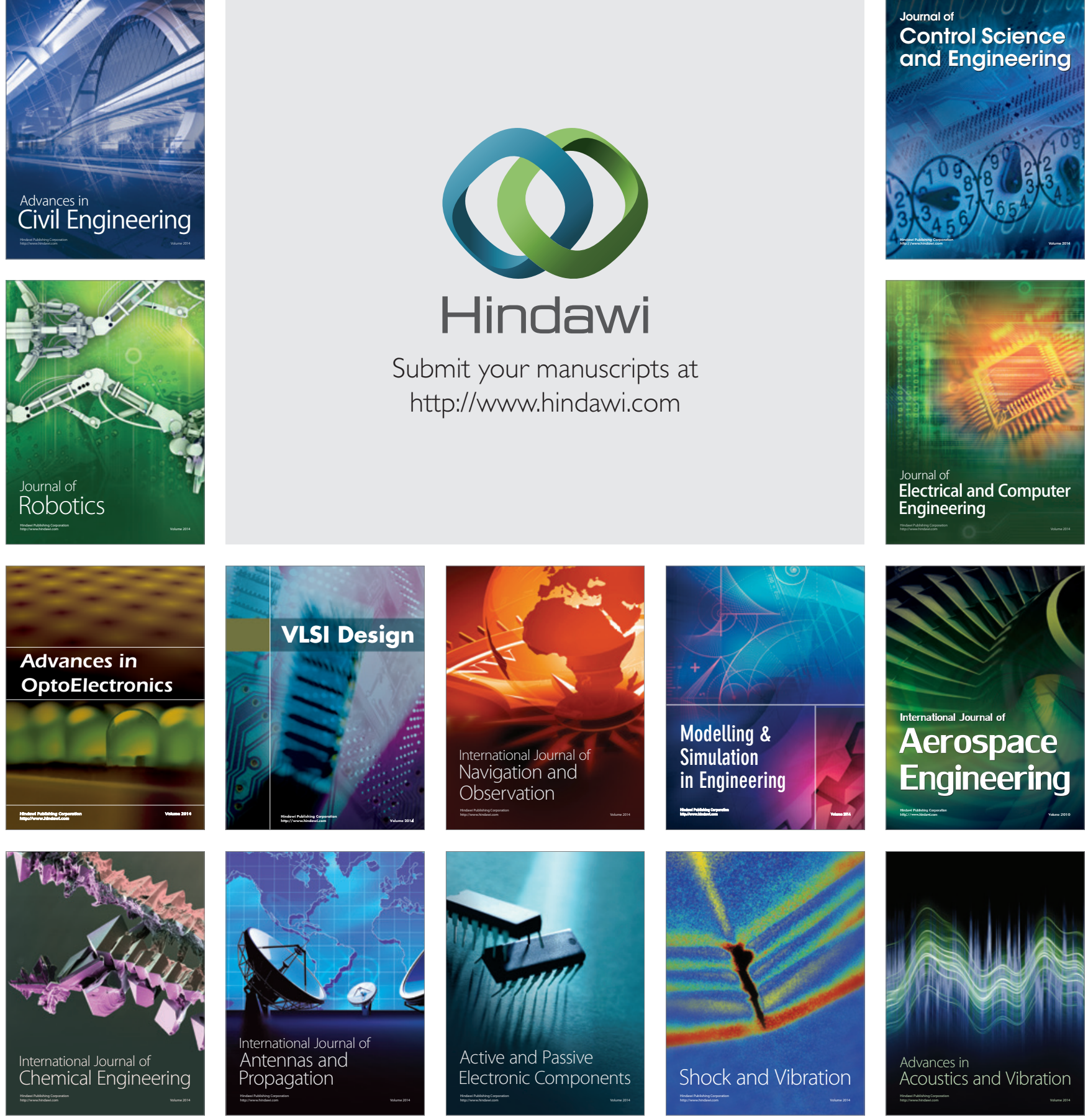